\title{
Graph Laplacians and Stabilization of Vehicle Formations
}

\author{
J. Alexander Fax Richard M. Murray \\ Engineering and Applied Science \\ California Institute of Technology \\ Pasadena, CA 91125 \\ $\{$ fax, murray\}@cds. caltech.edu
}

July 27,2001

\begin{abstract}
Control of vehicle formations has emerged as a topic of significant interest to the controls community. In this paper, we merge tools from graph theory and control theory to derive stability criteria for formation stabilization. The interconnection between vehicles (i.e., which vehicles are sensed by other vehicles) is modeled as a graph, and the eigenvalues of the Laplacian matrix of the graph are used in stating a Nyquist-like stability criterion for vehicle formations. The location of the Laplacian eigenvalues can be correlated to the graph structure, and therefore used to identify desirable and undesirable formation interconnection topologies.
\end{abstract}

\section{Introduction}

Recent years have seen the emergence of control of vehicle formations as a topic of significant interest to the controls community. Applications of this span a wide range, including mobile robotics, traffic control, satellite clusters and UAV formations. A recent study [1] identified this area as needing fundamentally new control paradigms.

Broadly speaking, this problem falls within the domain of decentralized control, but it possesses several unique aspects which are of note. This first is that vehicles in a formation are, as a rule, dynamically decoupled, meaning the motion of one vehicle does not directly affect any of the other vehicles. ${ }^{1}$ Instead, the vehicles are coupled through the task which they are jointly asked to accomplish. Tasks of this nature include requiring a formation to fly in a specific pattern, distribute itself evenly over a specified area, or arrive simultaneously at specified endpoints. Other tasks include the assignment of roles to individual vehicles within a formation which enable the entire formation to accomplish a higher-level task. When the formation is dynamically coupled, that coupling constrains, or at least naturally suggests, what information must be available to each component of the decentralized controller. In the case of cooperative vehicle control, no such architecture is necessarily suggested. As such, a second unique aspect of cooperative vehicle control is the fact that the interconnection structure between vehicles is not a given. It may be available as a design parameter, or the control architecture may require sufficient flexibility to handle changes in the interconnection structure.

\footnotetext{
${ }^{1}$ There are exceptions, of course, such as drag reduction via formation flight of airplanes, but we are not considering those in this study.
} 
We see that central to a discussion of cooperative vehicle control is a determination of the nature of the information flow throughout the formation. We will distinguish between two types of information flow: sensed information, meaning the ability of a single vehicle to sense some information (e.g. rrelative position) about another vehicle in a way which involves no action on the part of that vehicle, and transmitted information, meaning transfer of information between two vehicles which requires some action on the part of both the transmitter and recipient of that information. Sensing and transmission, or "seeing" and "hearing," together are the means by which each vehicle acquires the information necessary to perform its task within the formation.

Several observations about the information flow within a formation make clear the need to consider its impact on the formation performance. The first is that as a rule, no vehicle will be able to see or hear the entire formation. Thus, having each vehicle simultaneously solve a centralized control problem using complete information is infeasible, thus necessitating some form of decentralized control. The second observation is the sensing and transmitted information topologies are themselves dynamic, meaning they are subject to change due to changes in the formation itself or due to outside influences. As such, a control law which is optimized for one topology may exhibit poor performance, or even instability, for another topology.

A natural way to model the information flow topology is as a graph. Each vehicle is modeled as a node on the graph, and an arc joins node $i$ to node $j$ if vehicle $j$ is receiving information from vehicle $i$. To accommodate the full range of possible topologies, we will consider directed graphs, meaning bidirectional communication is not assumed. Several authors [4, 12] have used graphtheoretic ideas in control of vehicle formations. In both those papers, the formation implements a variant of a leader-follower architecture. In graph-theoretic terminology, such a formation is acyclic, meaning no sequence of arcs leads from a node back to itself.

Acyclic graphs are simpler to work with in that stability and performance analyses are local. However, they possess disadvantages which lead us to study the behavior of vehicle formations whose interconnection topology is not acyclic. Firstly, leader-follower architectures are known to have poor disturbance rejection properties (see, e.g., [14]). Secondly, enforcing an acyclic architecture requires some global knowledge of the graph, which may not be available to a given vehicle deciding which vehicle to follow. Finally, a leader-follower architecture depends heavily on the leader for achieving its goal, and over-reliance on a single vehicle in the formation may be undesirable, especially in adversarial environments.

For these reasons, we wish to consider a broader range of vehicle interconnection possibilities. The first challenge in this case is formation stabilization. When cycles are present in the graph, the dynamics of each vehicle are not purely local; an additional global component is present, which comes as a result of cycles in the graph. While the formation dynamics can be written down as a single system, such a representation is not useful for controller synthesis.

Our goal in this paper is to derive stability criteria for the formation which facilitate controller design on the local level. Central to this development will be the use of the Laplacian of the graph, a matrix representation of the graph whose spectral properties can be related to structural properties of the graph $[3,11]$. The Laplacian has been used previously in the study of chaos in interconnected oscillators $[9,13]$. In this paper, we take a control-theoretic approach to stability analysis of interconnected vehicles. For the problem of relative formation stabilization, we will present a Nyquist-like criterion for formation stabilization, and we use the spectral properties of the Laplacian to evaluate desirable structural properties of the graph. In a companion paper, [6], we will study techniques by which information can be shared between vehicles to improve stability margins and formation performance. 


\section{Problem Setup}

The problem we consider is the stabilization of a set of vehicles where only relative measurements are available to any given vehicle. Problems of this type include vehicle platoons [14] and satellite formations [15]. We consider a set of $N$ vehicles, whose (identical) linear dynamics are denoted

$$
\dot{x}_{i}=A x_{i}+B u_{i}
$$

where $i \in[1, N]$ is the index for the vehicles in the flock. Note that each vehicle's dynamics are decoupled from the vehicles around it. Each vehicle's sensed information is defined as follows:

$$
\begin{aligned}
y_{i} & =C_{1} x_{i} \\
z_{i j} & =C_{2}\left(x_{i}-x_{j}\right), j \in J_{i}
\end{aligned}
$$

Thus, $y_{i}$ represents absolute state measurements, and $z_{i j}$ represents relative state measurements. The set $J_{i} \subset[1, N] \backslash\{i\}$ represents the set of vehicles which vehicle $i$ can sense. It is assumed that $J_{i} \neq \emptyset$, meaning each vehicle can see at least one other vehicle. Note that a single vehicle cannot drive all the $z_{i j}$ signals to zero; the errors must be synthesized into a single signal. For simplicity, we will assume that all relative state measurements are weighted equally to form one relative measurement:

$$
z_{i}=\frac{1}{\left|J_{i}\right|} \sum_{j \in J_{i}} z_{i j}
$$

Of course, we could also weight different measurements differently. We also define a decentralized control law $K(s)$ which maps $y_{i}, z_{i}$ to $u_{i}$, represented in state-space form by

$$
\begin{aligned}
\dot{v}_{i} & =F v_{i}+G_{1} y_{i}+G_{2} z_{i} \\
u_{i} & =H v_{i}+J_{1} y_{i}+J_{2} z_{i},
\end{aligned}
$$

We now consider the system of all $N$ vehicles together. The hatted matrices, for example $\hat{A}$, represent the matrix $A$ repeated $N$ times along the diagonal. We can represent the total system as follows:

$$
\begin{aligned}
\dot{x} & =\hat{A} x+\widehat{B J_{1} C_{1}} x+\widehat{B J_{2} C_{2}} L x+\widehat{B H} v \\
\dot{v} & =\hat{F} v+\widehat{G_{1} C_{1}} x+\widehat{G_{2} C_{2}} L x
\end{aligned}
$$

or

$$
\left(\begin{array}{c}
\dot{x} \\
\dot{v}
\end{array}\right)=\left(\begin{array}{ll}
\hat{A}+\widehat{B J_{1} C_{1}}+\widehat{B J_{2} C_{2}} L_{(n)} & \widehat{B H} \\
\widehat{G_{1} C_{1}}+\widehat{G_{2} C_{2}} L_{(n)} & \hat{F}
\end{array}\right)\left(\begin{array}{l}
x \\
v
\end{array}\right) .
$$

The resulting system is block diagonal with the exception of the matrix $L_{(n)}$, which contains the relative sensing information. We now turn our attention to this matrix. Define $L$ in the following way:

$$
\begin{aligned}
L_{i i} & =1 \\
L_{i j} & =\left\{\begin{array}{l}
-\frac{1}{\left|J_{i}\right|}, j \in J_{i} \\
0, \quad j \notin J_{i}
\end{array}\right.
\end{aligned}
$$

Letting $n$ be the dimension of $x_{i}, L_{(n)}$ is of dimension $N n \times N n$ and is defined by replacing each element of $L$ with that element multiplied by $I_{n}$, thus generating a version of $L$ dimensionally compatible with $x_{i}$. 


\section{Laplacians of Graphs}

Before we consider the graph-theoretic aspects of $L$, we introduce some terminology. There are many introductory texts on graph theory; [5] is one. A directed graph $\mathcal{G}$ consists of a set of vertices, or nodes, denoted $\mathcal{V}$, and a set of $\operatorname{arcs} \mathcal{A}$, where $a \in \mathcal{A}=(v, w)$ and $v, w \in \mathcal{V}$. The first element of $a$ is denoted tail $(a)$, and the second is denoted the head $(a)$. It is said that $a$ points from $v$ to $w$. We will assume that $\operatorname{tail}(a) \neq \operatorname{head}(a)$, meaning that the graph has no loops. We also assume that each element of $\mathcal{A}$ is unique. A graph with the property that $(v, w) \in \mathcal{A} \Rightarrow(w, v) \in \mathcal{A}$ is said to be undirected, and the pair of arcs can be modeled as a single edge with no direction associated to it. The in-degree of a vertex $v$ is the number of arcs with $v$ as its head, and the out-degree is the number of arcs with $v$ as its tail. For an undirected graph, the in-degree and out-degree of a given vertex are equal. If all vertices have the same in(out)-degree, the graph is said to be in(out)-regular.

A path on $\mathcal{G}$ of length $N$ from $v_{0}$ to $v_{N}$ is an ordered set of distinct vertices $\left\{v_{0}, v_{i}, \ldots, v_{N}\right\}$ such that $\left(v_{i-1}, v_{i}\right) \in \mathcal{A} \forall i \in[1, N]$. An $N$-cycle on $\mathcal{G}$ is defined the same as a path except that $v_{0}=v_{N}$, meaning the path rejoins itself. A graph without cycles is said to be acyclic. A graph with the property that the set of all cycle lengths has a common divisor $M$ other than one is said to be $M$-periodic. A graph with the property that for any $v, w \in \mathcal{V}$, there exists a path from $v$ to $w$, is said to be strongly connected.

We now turn to matrices associated with graphs. For this purpose, we assume that the vertices of $\mathcal{G}$ are enumerated, and each is denoted $v_{i}$. The adjacency matrix of a graph, denoted $A$, is a square matrix of size $|\mathcal{V}|$, defined by $A_{i j}=1$ if $\left(v_{j}, v_{i}\right) \in \mathcal{A}$, and is zero otherwise. Note that $A$ uniquely specifies a graph. Let $D$ be the matrix with the in-degree of each vertex along the diagonal (assume each vertex has nonzero in-degree). The Laplacian of the graph is defined as ${ }^{2}$

$$
L=I-D^{-1} A
$$

Figure 1 shows an example of a directed graph, its arc set and its Laplacian. The Laplacian matrix is an object of study within graph theory. Specifically, the eigenvalues of the Laplacian can be related to structural properties of its graph. We now note some basic, well-known properties of the Laplacian $[3,11]$.

1. $0 \in \lambda(L)$, and the associated eigenvector is $1_{N \times 1}$. This can be proven from the fact that all rows of $L$ sum to zero.

2. The matrix $D^{-1} A$ is a nonnegative matrix. Thus, Perron-Frobenius theory (see, e.g., [10]) is a useful tool in analyzing spectral properties of the Laplacian.

3. All eigenvalues of $L$ are located in a disk of radius 1 in the complex plane centered at $1+j 0$. This can be shown by applying Gershgorin's theorem to the rows of $L$.

4. If $\mathcal{G}$ is strongly connected, 0 is an isolated eigenvalue of $L$. (The zero eigenvalue of $L$ corresponds to the Perron eigenvalue of $D^{-1} A$ at 1 . Strong connectivity of the graph corresponds to irreducibility of $D^{-1} A$.)

5. If $\mathcal{G}$ is aperiodic, then the modulus of any non-Perron eigenvalues of $D^{-1} A$ is strictly less than 1. if $\mathcal{G}$ is $M$-periodic, then $L$ has $M$ eigenvalues on the boundary of the Gershgorin disk with an angular spacing of $\frac{2 \pi}{M}$.

\footnotetext{
${ }^{2}$ Some references define $L$ as $D-A$. Also, some references use the out-degree in the definition of the Laplacian for directed graphs. The two are essentially equivalent, and our application is better suited to use of the in-degree.
} 


$$
\begin{aligned}
\mathcal{A}= & \{(2,1),(1,2),(3,2), \\
& (4,2),(1,3),(4,3),(1,4)\} \\
L= & \left(\begin{array}{rrrr}
1 & -1 & 0 & 0 \\
-\frac{1}{3} & 1 & -\frac{1}{3} & -\frac{1}{3} \\
-\frac{1}{2} & 0 & 1 & -\frac{1}{2} \\
-1 & 0 & 0 & 1
\end{array}\right)
\end{aligned}
$$

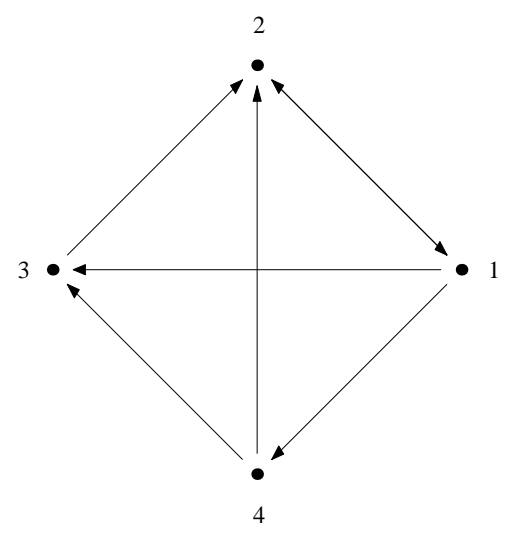

Figure 1: Graph, Arc Set and Laplacian Matrix

6. If $\mathcal{G}$ is an undirected graph, all eigenvalues of $L$ are real.

7. Because $L$ has ones along the diagonal, the trace of $L$ is $N$. Thus, the nonzero eigenvalues will tend to be clustered around $1+\frac{1}{N-1}$.

In the sections which follow, we will identify the role Laplacians play in formation stability analysis, and use the ideas mentioned above to evaluate the effects of certain formation interconnection structures on formation stability.

\section{Stabilization of Vehicle Formations}

We wish to consider the relationship between graph Laplacians and formation stabilization. We show the following to be true:

Theorem 1 A local controller K(s) stabilizes the formation dynamics in Equation (9) iff it simultaneously stabilizes the set of $N$ systems

$$
\begin{aligned}
\dot{x} & =A x+B u \\
y & =C_{1} x \\
z & =\lambda_{i} C_{2} x
\end{aligned}
$$

where $\lambda_{i}$ are the eigenvalues of $L$.

Proof We will show the above to be true by transforming the closed-loop dynamics in the following way: Let $T$ be a Schur transformation of $L$, meaning the unitary matrix such that $U=T^{-1} L T$ is upper triangular with the eigenvalues of $L$ along the diagonal [10]. Clearly, $T_{(n)}$ is a Schur transformation of $L_{(n)}$. This transformation has the following useful property, a clear consequence of the block structure of the relevant matrices: 
Lemma 1 Let $X$ be an $r \times s$ matrix, and $Y$ be an $N \times N$ matrix. Then

$$
\hat{X} Y_{(s)}=Y_{(r)} \hat{X}
$$

Applying this property to the system dynamics, we see if we let $\tilde{x}=T_{(n)} x$, and $\tilde{v}=T_{(m)} v$, we can rewrite Equation (9) as

$$
\left(\begin{array}{c}
\dot{\tilde{x}} \\
\dot{\tilde{v}}
\end{array}\right)=\left(\begin{array}{ll}
\hat{A}+\widehat{B J_{1} C_{1}}+\widehat{B J_{2} C_{2}} U_{(n)} & \widehat{B H} \\
\widehat{G_{1} C_{1}}+\widehat{G_{2} C_{2}} U_{(n)} & \hat{F}
\end{array}\right)\left(\begin{array}{l}
\tilde{x} \\
\tilde{v}
\end{array}\right) .
$$

The elements of the transformed system matrix are either diagonal or upper triangular. This means that stability of this system is equivalent to the stability of the systems along the diagonal, i.e.

$$
\begin{aligned}
\dot{\tilde{x}}_{i} & =\left(A+B J_{1} C_{1}+\lambda_{i} J_{2} C_{2}\right) \tilde{x}_{i}+B J_{2} C_{2} \tilde{v}_{i} \\
\dot{\tilde{v}}_{i} & =\left(G_{1} C_{1}+\lambda_{i} G_{2} C_{2}\right) \tilde{x}+F \tilde{v}
\end{aligned}
$$

which is equivalent to the controller $K(s)$ stabilizing the system

$$
\begin{aligned}
\dot{x} & =A x+B u \\
y & =C_{1} x \\
z & =\lambda_{i} C_{2} x
\end{aligned}
$$

In this context, the zero eigenvalue of $L$ can be interpreted as the unobservability of absolute motion of the formation in the measurements $z_{i}$. It seems that a prudent controller design strategy is to close an inner loop around $y_{i}$ such that the result system is stable, and then to close an outer loop around $z_{i}$ which achieves desired formation performance. For the remainder of this paper, we concern ourselves solely with the outer loop. Hence, we assume from now on that $C_{1}$ is empty and that $A$ has no eigenvalue in the open RHP. We do not wish to exclude eigenvalues along the $j \omega$ axis because many vehicle formations (e.g. vehicle platoons, satellite clusters) possess those, and the presence of unobservable secular or periodic motion of the formation may be tolerable in those cases. If $K(s)$ stabilizes the system in Equation (20) for all $\lambda_{i}$ other than the zero eigenvalue, we will say that it stabilizes the relative formation dynamics.

In general, proving simultaneous stabilization results can be difficult. This set of systems is special, in that they differ only by a complex scalar. For SISO systems, we can state a second version of Theorem 1 which is useful for stability and robustness analysis:

Theorem 2 Suppose $G(s)=C_{2}(s I-A)^{-1} B$ is SISO. Then $K(s)$ stabilizes the relative formation dynamics iff the net encirclement of $-\lambda_{i}^{-1}$ by the Nyquist plot of $K(s) G(s)$ is zero for all nonzero $\lambda_{i}$.

Proof The Nyquist Criterion states that stability of the closed loop system in Theorem 1 is equivalent to the number of CCW encirclements of $-1+j 0$ by the forward loop $\lambda_{i} K(j \omega) G(j \omega)$ being equal to the number of RHP poles of $G(s)$, which is assumed to be zero. This criterion is equivalent to the number of encirclements of of $-\lambda_{i}^{-1}$ by $K(j \omega) G(j \omega)$ being zero.

In the case where $G(s)$ is MIMO, the formation can be thought of as a structured uncertainty of the type scalar time identity (see [16]) where the scalars are the Laplacian eigenvalues. More 

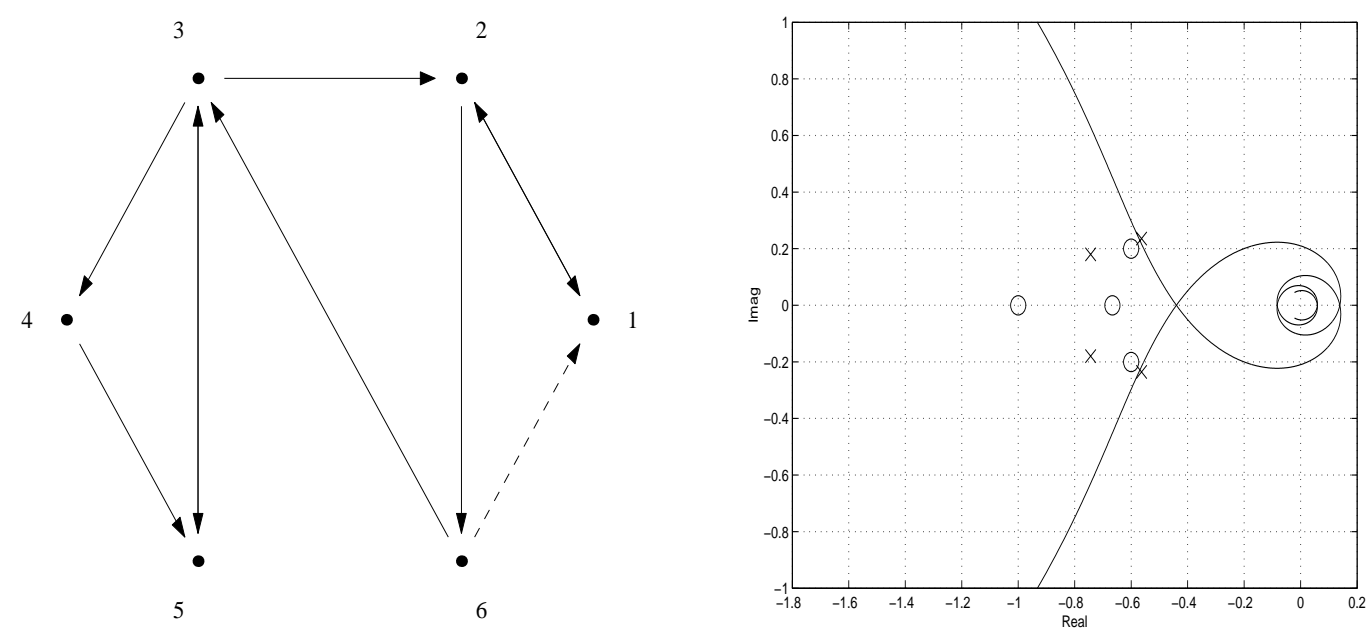

Figure 2: Formation Graph and Nyquist Plot

specifically, we shall write the eigenvalues as $\lambda_{i}=1+\mu_{i}$ and consider bounds on $\mu_{i}$. Suppose it is known that $\left|\mu_{i}\right| \leq M \forall \lambda_{i} \neq 0$. If we close the loop around the unity block and leave $\mu_{i} I$ as an uncertainty, resulting lower block is $C(s)=K(s) G(s)(I+K(s) G(s))^{-1}$, which is assumed to be stable. The following result from robust control theory then applies:

Theorem $3 K(s)$ stabilizes the MIMO formation $G(s)$ if

$$
\rho(C(j \omega))<M^{-1} \forall \omega \in(-\infty, \infty)
$$

Let us consider an example. Let $G(s)=\frac{e^{-s T}}{s^{2}}$ and $K(s)=K_{p}+K_{d} s$. This corresponds to a double integrator with a time delay being controller by a PD controller. Figure 2 shows a formation graph and the Nyquist plot of $K(s) G(s)$ with the Laplacian eigenvalues. The black 'x' locations in Figure 2 correspond to the eigenvalues of the graph defined by the black arcs in Figure 2, and the 'o' locations are for eigenvalues of the graph when the dashed arc is included as well. This example clearly shows the effect the formation has on stability margins. The standard Nyquist plot reveals a system with reasonable stability margins - about $8 \mathrm{~dB}$ and 45 degrees. When one accounts for the effects of the formation, however, one sees that for the ' $\mathrm{x}$ ' formation, the stability margins are substantially degraded, and for the 'o' formation, the system is in fact unstable. Interestingly, the formation is rendered unstable when additional information (its position relative to vehicle 6 ) is used by vehicle 1 . We shall return to this point shortly.

\section{$5 \quad$ Location of Laplacian Eigenvalues}

The location of Laplacian eigenvalues has emerged as the parameter which enables formation stability to be analyzed on the local level. A natural question to ask is: how does formation structure affect eigenvalue placement. We begin by considering simple formation structures and their eigenvalue placement. Examples of these graphs are shown in Figure 3.

1. Complete graph. The complete graph is one where every possible arc exists. In this case, the eigenvalues of a graph with $N$ vertices are zero and $1+\frac{1}{N-1}$, the latter repeated $N-1$ times. For large $N$, stabilization of the complete graph is equivalent to stabilizing an individual 

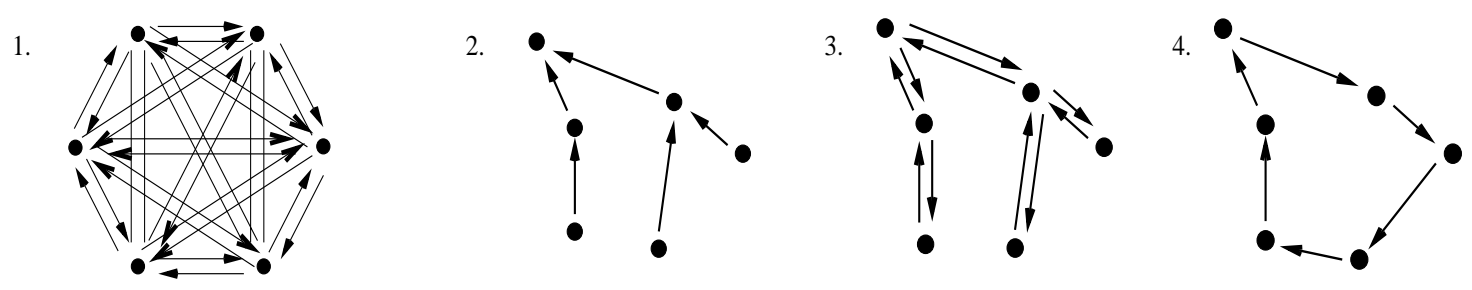

Figure 3: Sample Graph Structures

vehicle. Of course, a complete interconnection structure can place an enormous burden on each vehicle's sensing and computational capacities.

2. Acyclic (directed) graph. This graph has the 1 eigenvalue repeated $N$ times. This can be seen from the fact that the vertices can be ordered such that $L$ is upper triangular with ones along the diagonal. This is the 'leader-follower' architecture discussed in the introduction. In this case, vehicle stabilization is truly a local result, since other vehicles' dynamics enter only as a disturbance. However, this architecture has drawbacks, as discussed earlier.

3. Two-cyclic undirected graph. A graph of this type would include a vehicle platoon with bidirectional position measurement. This graph will have an eigenvalue at 2, due to its periodicity, and all other eigenvalues will be real, due to the symmetry of the graph.

4. Single directed cycle. This graph has eigenvalues at $e^{j(i-1) / 2 \pi}, i \in[1, N]$. These eigenvalues live on the boundary of the Gershgorin disc in which all the eigenvalues must lie.

Figure 4 shows various eigenvalue regions for $-L$ and the corresponding regions for $-L^{-1}$. The region bounded by the solid line is the Gershgorin disc in which all eigenvalues must lie. Its inverse is the LHP shifted by -0.5 . The dashed region is a bound in the magnitude of the nonzero eigenvalues of $L$. It corresponds to a shifted circle on the right hand side of Figure. Finally, the dash-dot line corresponds to a bound on the real component of the eigenvalues. The inverse of this bound corresponds to a circle which touches the origin.

Significant efforts have been made by graph theorists (see [3],[11], and references therein) to relate eigenvalue location to various graph-theoretic properties. These results were largely derived for undirected graphs, whose eigenvalues are real and can be bounded using variational techniques. For directed graphs, the problem is more challenging. Nonetheless, we can make qualitative statements about the effect of graph structure on eigenvalue placement, and hence stability.

If we consider the complete graph and the single directed cycle graph of Figure 3 as representing two extremes - one with all eigenvalues at a single location, the other with eigenvalues maximally dispersed, we see that eigenvalue placement can be related to the rate of mixing of information through the network. When the graph is highly connected, the global component of an individual vehicle's dynamics are rapidly averaged out through the rest of the graph, and thus has only a minor effect on stability. When the graph is periodic, the global component of the dynamics introduces periodic forcing of the vehicle, and the rest of the network never averages it out. This is represented on the Nyquist plot by putting the inverse eigenvalues nearer to the imaginary axis, thus degrading stability margins.

We see that aperiodicity is a desirable property of formation interconnection topologies. With this insight, we can see why the system in Figure 2 loses stability margin when a link is added. The 


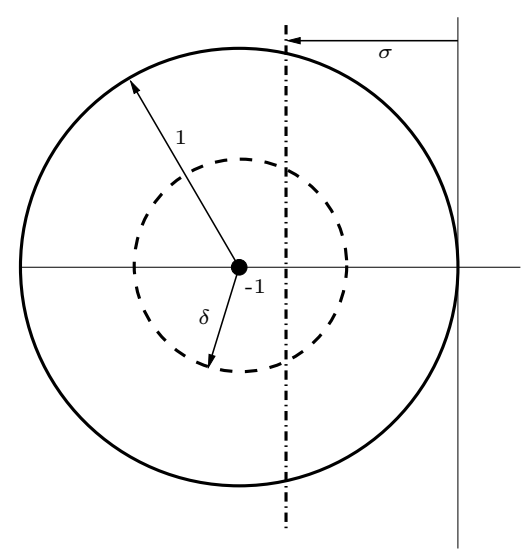

$\lambda(L)$

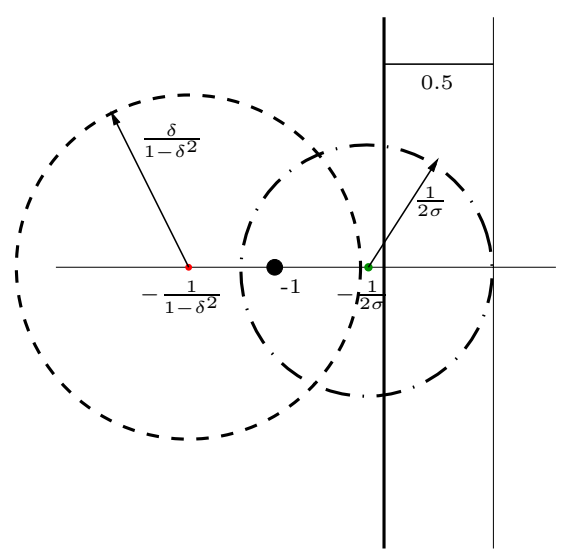

$\lambda\left(L^{-1}\right)$

Figure 4: Laplacian Eigenvalue Regions

'solid' graph possesses two 3-cycles and two 2-cycles. When the dashed link is added, an additional 3 -cycle is created, rendering the graph more nearly 3-periodic. This drives two of the eigenvalues nearer to the positions they would occupy if the graph were truly periodic, i.e., the -0.5 vertical.

Another aspect of of eigenvalue placement which deserves mention is that of weakly connected graphs. If we consider two unconnected graphs (two graphs with no arcs between either of them), then clearly the Laplacian has two eigenvalues at zero. If we weakly connect the graphs, one of the eigenvalues moves slightly away from zero. The smallest nonzero eigenvalue of a Laplacian is often seen as a measure of connectivity of a graph [7]. If the two graphs are themselves relatively connected when compared with the interconnection between them, this nonzero eigenvalue will be separated from the cluster of eigenvalues. These eigenvalues were used in [2] to define multiple time scales in sparsely connected networks, and their eigenvectors were used in [8] to split a graph into highly connected subgraphs. From a controls perspective, these eigenvalues represent weakly observable modes. On the formation Nyquist plot, they will be far from the origin, but they have the potential to destabilize a graph if its Nyquist plot possess an upper gain margin. These eigenvalues could be of significance when considering the merging or splitting of formations.

\section{Conclusions}

In this paper, we have presented a Nyquist-like criterion for assessing the effects of the interconnection topology on the stability of a formation of vehicles. The criterion is local, in that it is stated in terms of the dynamics of a single vehicle, and it gives insight into the effects of graph structure on stability. To be sure, many variants to the problem presented here could be presented (e.g. weakly connected graphs, mixed relative and absolute position objectives). Our goal in this paper is to motivate the role graph-theoretic ideas can have in formation controller analysis and design.

We have focused thus far on the flow of sensed information through the network. A natural topic of interest is how stability (as well as disturbance rejection and other measures of interest) can be improved through transmission of information between vehicles. In the extreme case, vehicles 
could share all information (assuming strong connectivity of the transmitted information graph), and each vehicle could essentially realize a centralized control law. However, this approach has obvious drawbacks in terms of bandwidth and computational complexity. In a companion paper [6], we will present strategies for sharing minimal amounts of information between vehicles, and how that information can be used to render the formation more robust to changes in the various topologies.

\section{References}

[1] S. Banda, J. C. Doyle, R. M. Murray, J. Paduano, J. Speyer, and G. Stein. Research needs in dynamics and control for uninhabbited aerial vehicles. Panel Report, November 1997. http://www.cds.caltech.edu/ murray/notes/uav-nov97.html.

[2] J.H. Chow and Petar V. Kokotovic. Time scale modeling of sparse dynamic networks. IEEE Transactions on Automatic Control, 30(8):714-722, August 1985.

[3] Fan R. K. Chung. Spectral Graph Theory, volume 92 of Regional Conference Series in Mathematics. American Mathematical Society, 1997.

[4] J. Desai, V. Kumar, and J. Ostrowski. A theoretical framework for modeling and controlling formations of mobile robots. IEEE Transactions on Robotics and Automations, 2001. under review.

[5] Reinhard Diestel. Graph Theory, volume 173 of Graduate Texts in Mathematics. SpringerVerlag, 1997.

[6] J.A. Fax and R. Murray. Information flow and vehicle formation control. in preparation.

[7] Miroslav Fiedler. Algebraic connectivity of graphs. Czechoslovak Mathematical Journal, 23:298-305, 1973.

[8] Miroslav Fiedler. A property of eigenvectors of nonnegative symmetric matrices and its applications to graph theory. Czechoslovak Mathematical Journal, 25(100):619-633, 1975.

[9] J.F. Heagy, T.L. Carroll, and L.M. Pecora. Synchronous chaos in coupled oscillator systems. Physical Review E, 50(3):1874-1885, September 1994.

[10] Roger Horn and Charles Johnson. Matrix Analysis. Cambridge University Press, 1985.

[11] Russell Merris. Laplacian matrics of graphs: A survey. Linear Algebra and its Applications, 197,198:143-176, 1994.

[12] M. Mesbahi and F. Hadaegh. Formation flying of multiple spacecraft via graphs, matrix inequalities, and switching. AIAA Journal of Guidance, Control and Dynamics, 24(2):369377, March 2001.

[13] L.M. Pecora and T.L. Carroll. Master stability functions for synchronized coupled systems. Physical Review Letters, 80(10):2109-2112, March 1998.

[14] Diana Yanakiev and Ioannis Kanellakopoulos. A simplified framework for string stability analysis in AHS. In Proceedings of 13th IFAC World Congress, volume Q, pages 177-182, San Francisco, CA, 1996. 
[15] Hsi-Han Yeh and Andrew Sparks. Geometry and control of satellite formations. In Proceedings of the American Control Conference, June 2000.

[16] Kemin Zhou and John Doyle. Essentials of Robust Control. Prentice Hall, New Jersey, 1998. 